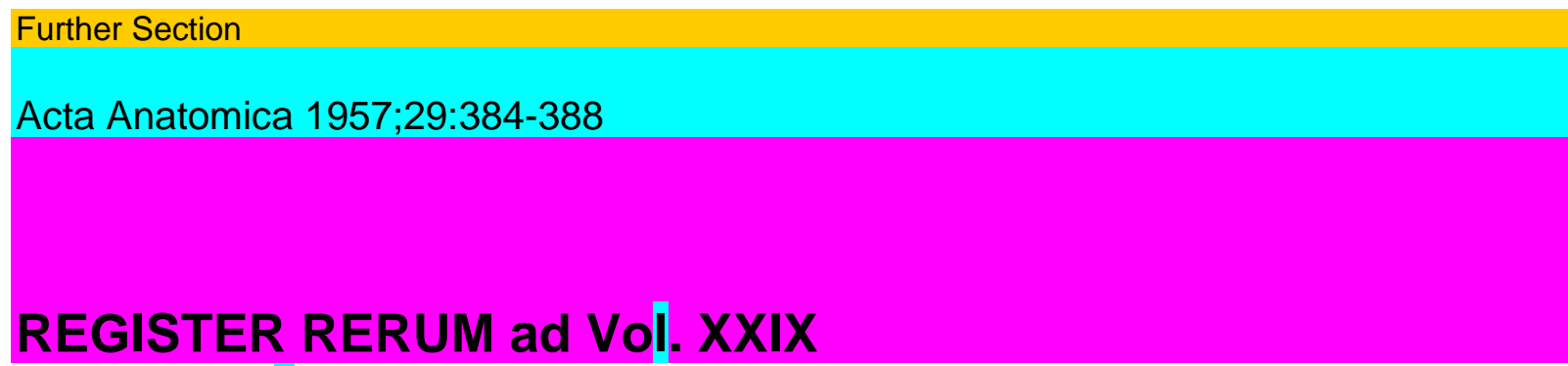

Confecit G. Boehm, Basel

Alveole $\pi$, v. Lymph system, Respiratory epithelium

Anatomie des Menschen, Lehrbuch (I. Bewegungsapparat) (B) 379

-, (II. Eingeweide und periphere Leitungsbahnen I) (B) 383

Anatomie, v. Pathologische Anatomie, Poumon, Topographische Anatomie

Arteria carotis, Arteria occipitalis, Artcria subclavia, v. Arteries

Arteriae vertebrales, v. Vascularisation

Arteries; on the possible honiologies of the occipital artery in mammals, with

some remarks on the phylogeny and certain anomalies of the subclavian and

carotid arteries $\quad 90$

Auris interna, v. Ductus cochlearis

Aves, v. Lymphoid tissue

Avitaminose, v. Malformations encéphaliques

Bewegungsapparat, v. Anatomie

Biological moving variate. (One type of bionormal frequency distributions)

Biological variability, v. Biological moving variate

Biometrik, v. Zahnbogen

Bionormal frequency distributions, one type. Biological moving variate . . 20

Biorhythm, v. Biological moving variate

Blood, v. Megakaryocyte

Blutplättchen, v. Megakaryocyte

Bronchioli, v. Respiratory epithelium

Bubalus buffalus, v. Büffel

Büffel; das Verhalten der Chromosomen beim Büffel

Buffalo, v. Büffel Buffle, v. Büffel

Canal cochléaire, v. Ductus cochlearis Canis famíliarís, v. Lymph system Carotid arteries, v.

Arteries

Cerebral cortex of the rat, development of a ground substance

Cerebrum; malformations encéphaliques par carence en acide pantothénique

et leur interpretation 209

-, v. Pathologische Anatomie, Subfornikales Organ Chicken, domestic, v. Lymphoid tissue

Chromosomen; das Verhalten der Chromosomen beim Büffel

323

Cobaye, v. Moelle épinière, Zahnkeime

Cochlea, v. Ductus cochlearis

Collagen, v. Kollagen

«Cornules incisives» du lièvre (Lepus europaeus Pallas), histogénèse et histo-

logie 330

Cortex, cerebral, of the rat, development of a ground substance

297

Cortisches Organ, v. Ductus cochlearis Cultures de tissu, v. Epithelkulturen 
(B) $=1$ Book reviews - Livres nouveaux - Buchbesprechungen

$(\mathrm{R})=$ Survey - Revue générale - Übersichtsreferat

Register rerum

385

Cytochemistry, v. Ground substance

Dentes, v. Zähne, Zahnkeime Development, v. Ground substance Diaphragma, v. Diaphragme

Diaphragme humain, presence de fuseaux neuro-musculaires

114

Ductus cochlearis; zur Kenntnis der Differenzierungsvorgänge im Epithel des

Ductus cochlearis

53

Ear, v. Ductus cochlearis Eingeweide, v. Anatomie

Elektronenmikroskop, v. Kollagene Fibrillen, Megakaryocyte

Embryologische Beobachtungen an den Harnorganen der Maus und des Goldhamsters

Epithel des Ductus cochlearis, Differenzierungsvorgänge

Epithelial cells; respiratory epithelium

Epithelkulturen aus Nierenpapillen röntgenbestrahlter junger Mäuse, Untersuchung 236

Fibrillen, kollagene, Morphogenese (R)

Fine structure of the megakaryocyte in the mouse spleen

- , v. Kollagene Fibrillen

Freeze-drying, v. Gefriertrocknung

Frequency distributions, bionormal, one type. Biological moving variate . .20

Fuseaux neuro-musculaires dans le diaphragme humain

114

Gallus domesticus, v. Lymphoid tissue

Gaussian distribution, v. Biological moving variate

Gefäße, v. Arteries

Gefäßversorgung, v. Vascularisation

Gefriertrocknung, Grundriß (B) 296

Gehòrorgan, v. Ductus cochlearis

Gesicht, Breite; Beziehungen zwischen der Breite des Gesichtes, des Zahn-

bogens und der Zähne 117

Glucoprotein, v. Ground substance Goldhamster, v. Harnorgane

Ground substance in the cerebral cortex of the rat, development

Grundsubstanz, v. Ground substance Guinea-pig, v. Cobaye

Hamster, golden hamster, v. Harnorgane

Hare, v. Lièvre

Harnorgane; embryologische Beobachtungen an den Harnorganen der Maus

und des Goldhamsters

1

Hase, v. Lièvre

Hen, v. Lymphoid tissue

Histochemistry, v. Ground substance

Histologie, v. Istologia

Histologie, pathologische, spezielle, v. Pathologische Anatomie

Historie, v. Leonardo da Vinci

Homeostasis, v. Biological moving variate

386 


\section{Register rerum}

Homology, v. Arteries, Paraphyse Huhn (Haushuhn), v. Lymphoid tissue

Implantation, v. Zahnkeime

«Incisive horns» of the hare, v. Lièvre

Innenohr, v. Ductus cochlearis

Intestina, v. Anatomie

Isotope, radioaktive, $v$. Zahnkeime

Istologia, trattato (Vol. I und II) (B) 379

Jaws, v. Kieferbreite

Kidney, v. Epithelkulturen, Harnorgane

Kieferabdrücke, v. Kieferbreite

Kieferbreite; Beziehungen zwischen der Breite des Gesichtes, des Zahnbogens

und der Zähne 117

Kollagene Fibríllen, Morphogenese (R)

Leonardo da Vinci als Anatom (russisch) (B)

Lepus europaeus, v. Lièvre

Lièvre; les «cornules incisives» du lièvre (Lepus europaeus Pallas), histogénèse

et histologie 330

Limbus spiralis, v. Ductus cochlearis

Lunge, v. Lymph system, Poumon, Respiratory epithelium

Lymph system, pulmonary, origin 228

Lymph vessels, v. Lymphoid tissue

Lymphoid nodules, v. Lymphoid tissue

Lymphoid tissue, association with the lymph vessels in the domestic chicken

(Gallus domesticus) $\quad 36$

Mâchoire, v. Zahnbogen

Malformations encephaliques par carence en acide pantothénique et leur

interpretation 209

Mammalia, v. Arteries

Man, v. Anatomie, Arteries, Diaphragme, Pathologische Anatomie, Poumon,

Respiratory epithelium, Subfornikales Organ, Zahnbogen Mandibula, v. Zahnbogen Maxilla, v.

Zahnbogen Medulla spinalis, v. Vascularisation Meerschweinchen, v. Cobaye

Megakaryocyte in the mouse spleen, fine structure 267

Membrana tectoria, v. Ductus cochlearis

Memoranda 376

Mesocrícetus auratus, v. Harnorgane M«tanephros, v. Harnorgane Methode, v. Gefriertrocknung

Mißbildungen, v. Malformations encephaliques

Moelle épinière du cobaye, vascularisation 314

Morphogenese der kollagenen Fibrille (R) 143

Mouse, v. Mus musculus

Moving variate, biological. (One type of bionormal frequency distributions.)

Mucopolysaccharide, v. Ground substance

Register rerum 387

Mucoprotein, v. Ground substance

Muko ..., v. Muco ...

Mus musculus, v. Ductus cochlearis, Epithelkulturen, Harnorgane, Mega- 
karyocyte Muscle, v. Muskelfaser Muscle spindles, v. Diaphragme

Muskelfaser, quergestreifte, morphologische Veränderungen durch Ultraschall 344 Muskulatur

(Skeletmuskulatur), v. Anatomie

Nephren, v. Harnorgane

Nerven (Nervensystem, Zentralnervensystem), v. Anatomie, Cerebral cortex,

Cerebrum, Fuseaux neuro-musculaires, Moelle épinière, Pathologische

Anatomic, Subfornikales Organ Nierenbecken, Nierenkelch, v. Harnorgane Nierenpapillen, v.

Epithelkulturen

Occipital artery in mammals, possible homologies, with some remarks on the

phylogeny and certain anomalies of the subclavian and carotid arteries $\quad 90$

Ohr, v. Ductus cochlearis

Ontogenesis, v. «Cornules incisives», Ductus cochlearis, Harnorgane, Malformations

encéphaliques, Paraphyse

Oreille, v. Ductus cochlearis

Organum subfornicale, v. Subfornikales Organ

304381

383

228

Pantothensäuremangel, v. Malformations encéphaliques Papilla basilaris, v. Ductus cochlearis Paraphyse und subfornikales Organ, zur Frage ihrer Homologie

PAS-technique, v. Ground substance

Pathologische Anatomie und Histologie, spezielle (XIII. Bd., 4. Teil: Zen

tralnervensystem) (B)

Periodic acid (Schíff) (= PAS) technique, v. Ground substance

Phylogenesis, v. Arteries

Platelets, v. Megakaryocyte

Porionbreite, v. Kieferbreite

Poule, v. Lymphoid tissue

Poumon; les pédicules segmentaires du poumon (II.: Poumon gauche) (B)

-, v. Lymph system, Respiratory epithelium

Probability, v. Biological moving variate

Protein, v. Ground substance

Pulmo, v. Poumon, Respiratory epithelium

Pulmonary lymph system, origin

Radiation, v. Epithelkulturen

Rana, v. Subfornikales Organ, Ultraschall

Rat, v. Cerebral cortex, Malformations encéphaliques

Ren, v. Epithelkulturen, Harnorgane

47236

Renal calyx, Renal pelvis, v. Harnorgane

Respiratory epithelium

Röntgenbestrahlte junge Mäuse, Untersuchungen an Epithelkulturen aus

ihren Nierenpapillen

388

Register rerum

Rückenmark, v. Vascularisation 
Sclavounos, Georges, 1867-1954

Segmenta pulmonis, v. Poumon

Skelet, v. Anatomie

Skeletmuskel, v. Muskelfaser

Souris, v. Mus museulus

Spinal cord, v. Vascularisation

Splen, v. Megakaryocyte

Statistische Auswertung, v. Biological moving variate, Zahnbogen

Strahlenwirkung, v. Epithelkulturen

Stria vascularis, v. Ductus cochlearis

Subclavian arteries, v. Arteries

Subfornikales Organ und Paraphyse, zur Frage ihrer Homologie 304

Submicroscopical structure, v. Kollagene Fibrillen, Megakaryocyte Sulcus spiralis externus

(internus), v. Ductus cochlearis

Teratologie, v. Malformations encéphaliques Thrombocytes, v. Megakaryocyte Tissue cultures, v. Epithelkulturen

Topographische Anatomie, Lehrbuch (B) $\quad 380$

Tragionbreite, v. Kieferbreite

Transplantation von Zahnkeimen (III). (Nachweis des Anschlusses der Trans-

plantate an den Blutkreislauf des Wirtes mit Hilfe von Isotopen.) . . 291

Ultraschall; morphologische Veränderungen an der quergestreiften Muskel

faser durch Ultraschall 344

Ultrasonics, v. Ultraschall Ureteren, v. Harnorgane Urin, v. Harnorgane Urinary bladder, v.

Harnorgane

Vaisseaux, v. Arteries, Vascularisation Variability, v. Biological moving variate

Vascularisation de la moelle épinière du cobaye $\quad 314$

Vessels, v. Arteries, Vascularisation

Vinci, Leonardo da, als Anatom (russisch) (B) 381

Vitamin (Pantothensäure), v. Malformations encéphaliques

Wahrscheinlichkeit, v. Biological moving variate

X-rays, v. Epithelkulturen

Zähne; Beziehungen zwischen der Breitc des Gesichtes, des Zahnbogens und

der Zähne 117

Zahnbogen; Beziehungen zwischen der Breite des Gesichtes, des Zahnbogens

und der Zähne 117

Zahnkeime, Transplantation (III). (Nachweis des Anschlusses der Trans-

plantate an den Blutkreislauf des Wirtes mit Hilfe von Isotopen) . . 291

Zellkulturen, v. Epithelkulturen

Zwerchfell, v. Diaphragme

Zygionbreite, v. Kieferbreite 